\title{
Qui dit sang? Ou comment représenter autrement le sang féminin
}

\section{Dominique Bourque}

Some feminist authors and artists propose original representations of blood to counteract polarized gender depictions of this vital fluid in Western Culture. This article focuses on their use of history, utopia and metaphor to break myths and prejudices.

Signe de violence, de maladie, ou, plus rarement, d'espoir et de vie, on a fait du sang notre " pain quotidien ». Donné ou vendu, disponible non traité ou $100 \%$ pur, servi « gore " ou gothique, il ajoute désormais du piquant aux films hollywoodiens, de la couleur aux couvertures des best-sellers et de la saveur aux journaux à grand tirage. Façon de masquer celui qui coule dans les territoires occupés ou entre les jambes de petites filles du monde entier; qui gicle des corps d'enfants soldats ou sur les grabats de bordels et de prisons; qui macule les ruelles des bidonvilles ou les draps de salles d'opération clandestines? C'est Jésus qui disait: "Buvez-en tous, car ceci est mon sang, le sang de la nouvelle alliance, répandu pour la multitude en rémission des péchés » (Nouvelle Evangile 26: 26-29).

Face à la sempiternelle polarisation en fonction des sexes sociaux (genders) des représentations de cette " humeur fondamentale » ( There is a contrast of female association of blood as health and fertility versus male association of blood with illness and injury »Huerta), je m'intéresserai aux traitements originaux que nous en proposent des auteures et des artistes " féministes » - parfois avant la lettre! - en recourant à l'histoire, à l'utopie ou à la métaphore.

\section{Le Sang perdu}

Parmi les ouvrages de référence féministes, plusieurs renvoient au mot " menstruation " ${ }^{1}$. C'est le cas du Woman's Encyclopedia of Myths and Secrets de Barbara G. Walker - livre qui tente de démystifier nombre de figures symboliques en mettant au jour leurs sources. On y apprend par exemple que " Adam [comes] from the feminine adamath, meaning "bloody clay", though scholars more delicately translate it "red 
earth" » (Walker 63). L'origine étymologique du mot « Adam » resitue ainsi la conception du premier homme sur un plan beaucoup plus terre-à-terre que celle consignée dans la Bible. Voilà le mythe de l'homme " tout puissant " déboulonné sur l'autel du savoir, celui-là qui fut interdit aux femmes!

Dans un tout autre style, le Feminist Dictionary de Cheris Kramarae et de Paula A. Treichler propose trois notices sur le sang, toutes reliées aux menstrues. À la notice " menstruation », on découvre une remarque révélatrice à la fois du point de vue dominant (masculin ou masculiniste?) et de l'importance qu'accordent les féministes aux menstruations dans leurs écrits:

Studies of male scientists and physicians portrays it not only as a symptom of a disease but itself 'a morbid state fraught with danger' and 'an infirmity' which severely limits women's activities. But the evidence is overwhelming that women in modern society have always performed physical and other kinds of labor. (Kramarae et Trechler 76)

On apprend en outre qu'un certain docteur Edward H. Clark publia, au XIX siècle, un livre dans lequel il recommande que les filles étant donné l'ampleur des dommages que leur causent leurs règles ! passent moins de temps à étudier que les garçons de sorte qu'elles puissent se reposer trois ou quatre jours tous les mois.

Deux ouvrages plus récents me semblent également dignes de mention parmi ceux qui s'attaquent au mythe des règles débilitantes associées à une " nature féminine " (faiblesse, passivité, instabilité et capacités intellectuelles réduites). Dans Woman: An Intimate Geography (1999), Natalie Angier adopte, à la manière de Simone de Beauvoir dans Le Deuxième Sexe, une approche rigoureusement physiologique de la question. Toutefois, elle se méfie, contrairement à de Beauvoir, de la théorie de la nidification de l'utérus qui ramène les femmes à leur appareil reproducteur et cite la thèse controversée de la biologiste Margie Profet qui attribue aux menstruations une fonction principalement préventive.

Le deuxième ouvrage, The Frailty Myth (2000) de Colette Dowling, défend la thèse postulant qu'il n'existe pas de différences significatives entre les sexes. L'auteure y démolit, entre autres, l'idée fort répandue d'une fragilité accrue des femmes pendant leurs règles en rappelant l'exploit d'Uta Pipping. Cette athlète allemande gagna, en 1996, le marathon de Boston pour la troisième fois d'affilée - une première 
dans l'histoire de cet événement. Or elle le fit alors même qu'elle souffrait de crampes et d'une forte diarrhée, à la suite du déclenchement de ses menstruations au vu et au su de tout le monde.

La couverture médiatique malaisée que reçut l'événement allait dans un sens étonnant: l'athlète s'était couverte de ridicule. Il est encore « entendu " aujourd'hui que les individus de sexe féminin doivent avoir honte de perdre du sang tous les mois. Vouloir gagner une course importante, briser un record mondial, aller au bout de ses capacités, cela n'est pas "féminin ". Il faut qu'une femme soit " immaculée ", c'est-à-dire qu'elle cache son sang, trace du péché originel.

En approfondissant leurs connaissances dans les domaines de la linguistique, des sciences et de l'histoire, entre autres, des féministes ont clairement montré le caractère construit des perceptions que véhiculent les sociétés occidentales en ce qui concerne le sang menstruel (stigmate, saleté, signe de faiblesse et d'une plus grande proximité à la nature, etc.). Mais toutes n'ont pas adopté cette approche. Plusieurs ont également choisi d'entrer dans l'arène en investissant le domaine de l'imaginaire.

\section{Le Sang mythique}

On retrouve deux grandes catégories de figures de femmes dans les mythes que nous a légués l'Antiquité. Ils les représentent soit comme des êtres autonomes (chasseresses, combattantes, athlètes), soit comme des victimes. Outre les déesses, les premières appartiennent à la marge: il s'agit de rebelles que l'on finit par dompter, dominer, domestiquer. Ainsi, les Amazones, ces guerrières surnommées «les mangeuses d'hommes ", sont vaincues par Héraclès, Thésée, Achille et même "l'efféminé " Dionysos! Médée, la sorcière qui tue entre autres ses fils après que leur père l'eut abandonnée, doit s'exiler. Et les chasseresses d'Artémis sont constamment harcelées et traquées par les dieux et les hommes. Quant aux victimes, elles sont injustement emportées par la mort, cruellement violées ou blessées. Il en va ainsi d'Eurydice, la jeune épouse du poète Orphée, qui meurt d'une morsure de serpent; de Philomèle qui est violée et mutilée par son beaufrère, le roi de Térée (il lui coupe la langue); de Procris qui est transpercée par le javelot de son amant, de la prophète Cassandre qui ne fut jamais écoutée, assista impuissante au massacre de sa ville, fut retenue prisonnière et finalement tuée; de la superbe Niobé qui voit sa fierté punie par le massacre de ses enfants. Autrement dit, sois belle et taistoi, ou ne sois pas. 
De nombreux spécialistes des deux sexes s'accordent pour dire que ces mythes racontent, en filigrane, l'histoire de l'imposition de la culture patriarcale, en l'occurrence celle des envahisseurs achéens et doriens, à des cultures qui auraient vénéré de grandes déesses-mères dispensatrices de la vie comme de la mort.

Bien que le mythe de la " mangeuse d'hommes " n'ait pas disparu, il s'est radicalement transformé, puisque la « croqueuse d'hommes » ou "vamp " (abréviation de vampire) d'aujourd'hui apparaît relativement inoffensive. En ce sens, l'oblitération du caractère agressif des figures les plus indépendantes ainsi que la promotion d'une soi-disant douceur innée des femmes, d'un supposé pacifisme " naturel ", facilement assimilables à la passivité et à la fragilité, masqueraient une volonté de contrôle.

Mais les choses pourraient être plus compliquées, car si on en croit Jane Caputi, les grandes déesses auraient été promues Muses et propulsées à l'avant-scène, au début du $X X^{\mathrm{e}}$ siècle, par certains érudits pour contrer un penchant homosexuel chez les hommes.

For Graves [l'auteur du célèbre ouvrage The White Goddess], woman therefore occupies the position of symbol rather than subject, bearer rather than creator of meaning. Graves makes it apparent that this symbolic role is for men; the Muse exists to regulate men's relations with each other, not in her own right. It also becomes clear that the truth-claims of myths as the hidden secret repressed by civilization, are written by Graves as truths about femininity for men. (443)

Néanmoins, les quelques figures de femmes puissantes représentées dans les mythes et dans la Bible vont inspirer les premières peintres. Comme plusieurs de ses contemporains, Artemisia Gentileschi (15931652/3), par exemple, a représenté la scène de l'Ancien Testament où Judith décapite le roi des Assyriens, Holopherne, afin de sauver son peuple. Toutefois, ses toiles (elle a repris le sujet maintes fois) se distinguent clairement de celles de ces confrères, incluant celui qui l'a le plus influencée, à savoir Caravage. Bien que naturaliste, le célèbre peintre représente en effet une Judith beaucoup trop faible et féminine pour être crédible.

Only Gentileschi's masterful Judith Beheading Holoferns tells a realistic story. In this large canvas, the nearly life-size Judith is depict- 
ed as a powerful, determined woman, amply endowed with the physical and emotional strenght necessary to separate the head of a well-muscled general from his body. [...] This is a dirty business, and Gentileschi shows it: blood spurts everywhere, dripping obscenely down the sheets, adding a further note of realism to the dramatically spotlit scene. (Heller 32)

Dès lors, il devenait possible de représenter des personnages historiques, ce que fit Elisabetta Sirani (1638-1665) - qui connaissait également les figures mythologiques et bibliques -, lorsqu'elle a peint Porcia en train de se poignarder calmement la cuisse, sang à l'appui (1664).

La voie est désormais ouverte non seulement à l'appropriation de toutes les figures sacrées et marquantes, mais également à leur transgression. Ce sera le cas entre autres de l'auteure française Monique Wittig, qui subvertira, au début des années 70 dans Le Corps lesbien, le mythe de l'Eucharistie. Elle le fera en transformant le corps du Christ en corps lesbien, c'est-à-dire en remplaçant un corps de victime en un corps de désir, une passion douloureuse/sacrificielle en une passion jouissive/réciproque. D'où cette saisissante description d'une aimée trop longtemps « saignée ", enfin ressuscitée par la force des mots:

Tu es exsangue. [...] [T]on éclat efface le soleil. [...] Chaque goutte de ton sang chaque jaillissement de tes artères frappant $\mathrm{m} / \mathrm{es}$ muscles $\mathrm{m} / \mathrm{e}$ résonnent tout au long. $\mathrm{J} / \mathrm{e}$ ne peux pas bouger, $\mathrm{j} / /$ attends une apothéose une fin glorieuse dans ce lieu où les couleurs fondamentales ne font pas défaut, $\mathrm{j}$ /e tremble devant les rouge clair issus de tes artères, $\mathrm{j} / \mathrm{e}$ les vois virer au noir dans les taches tout autour de toi et sur m/on corps s'asséchant, $\mathrm{j} / \mathrm{e}$ regarde le sang sombre sortir du bleu de tes veines, par endroits il est violet figé, $[. ..] \mathrm{j} / \mathrm{e}$ ne te cherche pas $\mathrm{m} / \mathrm{a}$ vie, $\mathrm{j} / \mathrm{e}$ te suis là tout auprès [...]. (Wittig 13-14)

Trois années plus tard, Monique Wittig poursuit, cette fois avec l'auteure américaine Sande Zeig, son travail de déconstruction parodique des mythes en revalorisant des figures de femmes se nourrissant de sang: "Parmi les amazones et les sorcières, certaines ont eu la réputation d'avoir le privilège du sang. Ce sont les goules, les vampires, les lamies, les sirènes "(Wittig et Zeig 212). Les héroïnes faibles et anémiques acquièrent ainsi force et vivacité.

$\mathrm{Au}$ Québec, Louky Bersianik revoit également le mythe du 
"Sauveur ", mais en évoquant le messager justicier, comme en témoigne l'extrait suivant où l'Eugélionne dénonce les résistances des institutions face à l'avortement.

Vouloir du sang neuf à tout prix, en quantité illimitée, en quantité non contrôlée, sans tenir compte des structures Humaines déjà acquises, surpeupler une planète au risque de la voir éclater, préférer l'état léthargique d'un embryon sans désirs au désir de la femme de s'en débarrasser, ne pas pousser les recherches biologiques en survie et en gérontologie, sacrifier l'individualité des femmes à la reproduction de l'espèce; puis, envoyer ce sang neuf se faire répandre en terre étrangère au nom de la loi du plus fort, telle est la logique Humaine [...]. (Bersianik 270)

Miroirs, instruments d'analyse des sociétés et de leurs conditions de vie, tremplins vers des utopies ludiques, les mythes ont permis aux féministes occidentales de réévaluer la " réalité ", de mettre en relief le caractère construit de cette dernière afin d'en jouer. À l'heure de l'appropriation et de la recréation du texte mythique, il s'est agi d'opérer un retournement des perspectives, de proposer une " nouvelle alliance " qui tienne compte des femmes et de leur bien-être, car le premier corps sacrifié et exploité à travers le monde reste encore le leur ${ }^{2}$.

\section{Le Sang-froid}

Les femmes, dit-on, n'aiment ni le sang ni la violence. Et pourtant dans nombre de foyers de la planète, ce sont les mères de famille qui tuent la poule, éventrent le poisson, font boucherie et apprêtent la viande qui se retrouvera sur la table, ce sont des femmes qui excisent les petites filles. Jeanne d'Arc fut loin d'être la seule à revêtir l'armure pour aller se battre, bien d'autres s'engagèrent comme mercenaires à travers les âges ${ }^{3}$. On s'étonne des blessures qu'a infligées Valerie Solanas à Andy Warhol ou de l'existence de la tueuse en série Aileen Carol Wournos. Sait-on même qu'en l'an 2000, « quarante-sept femmes attend[ai]ent d'être exécutées dans les corridors de la mort américains " comme l'écrit Pierre Luc dans «Plus de pitié pour les meurtrières "? Mais s'il y a plus de criminelles aujourd'hui ${ }^{4}$, il y a également plus de chasseuses, de bouchères, de femmes médecins légistes, d'embaumeuses, etc.

Depuis une vingtaine d'années, les héritières d'Agatha Christie ne 
se comptent plus. Il suffit de consulter la section réservée aux romans policiers de n'importe quelle librairie. Les « reines du crime " portent des noms comme Patricia Highsmith, P. D. James, Ruth Rendell, Sandra Scoppettone, Gertrude Stein, Maud Tabachnik, Fred Vargas ou Helen Zahavi. Ces incursions audacieuses dans les chasses gardées masculines ne sont pas l'apanage des littéraires, et les artistes visuelles s'y adonnent elles aussi depuis déjà longtemps. Si Rosa Bonheur (18221899) réussit non seulement à s'imposer comme peintre animalier, mais à acquérir le titre de " world's greatest animal painter» (Heller 90), c'est, entre autres, parce qu'elle n'hésita pas à disséquer des pièces de viande et à visiter les abattoirs - ainsi qu'à faire renouveler tous les six mois son permis de port du pantalon!

Moins célébrée de son temps mais de plus en plus respectée, Frida Kahlo (1910-1954) annonce, quant à elle, la culture dite postmoderne dans la peinture en accordant une attention soutenue au corps humain morcelé et mis à vif, écorché:

All the latest imagery trends make use of human body: performers, visual artists, cyberartists, creatives, everybody uncovers bodies, opens bodies, cuts bodies, paints bodies, tattoos bodies. Fragments of bodies are spread everywhere: mere brains in magazines and tech ads, heads in S/W logos, hearts in Benetton's ads... Frida Kahlo made a little bit of all this 60 years ago, showing in her paintings. (Falini)

La robe de viande ${ }^{5}$ de la Canadienne d'origine tchèque Jana Sterbak s'inscrit dans cette tradition, de même que les œuvres extrêmement troublantes de l'anglaise Sue Coe. Issue de la classe ouvrière, celle-ci aborde directement la souffrance des démunis et des floués. Dans l'un de ses tableaux, on voit une main activer un hachoir à viande qui broie non pas un morceau de bœuf, mais un pied humain (Untitled, 1997). Dans un autre tableau, c'est un viol collectif qui est représenté ou la crucifixion d'un animal de laboratoire. Cette artiste qui n'a pas peur de qualifier son art de politique a également produit un livre intitulé Dead Meat Book dans lequel elle écrit: " Every dollar I get drips with blood too. " Or, aucun éditeur n'a d'abord voulu de cet ouvrage: « What I get is, "Your work's too strong." And I'm saying, "Well, is it supposed to be weak? Do you want weak work? Do you want work that's not about the truth? What are you telling me? »(Brant). Et on s'étonne de ne pas trouver de perspectives dissidentes, de points de vue autres sur le monde? 
Dans un tout autre registre, les autoportraits de la photographe américaine Catherine Opie participent également de cette culture postmoderne qui s'attarde au corps et à ses souffrances. Mais on assiste ici à un renversement, car les blessures ne sont plus seulement montrées, mais auto-infligées ou commandées. Dans Self Portrait/Cutting (1993), l'artiste, qui affiche son lesbianisme et son appartenance à la communauté « S/M ", apparaît de dos et nue devant une tenture damassée vert bouteille. Entre les omoplates, une main a gravé dans la peau des fillettes se tenant par la main, une petite maison avec une cheminée et un soleil à moitié caché par un nuage. Des gouttes de sang s'échappent des traits naïfs. Par cette œuvre, Catherine Opie voulait rendre visible la douleur intérieure qui la rongeait et tenter de la mater: " [It] was really out of losing a relationship, of breaking up after I finally thought I was going to have a home. I had really desired that " (Furguson 46).

L'anonymat de l'autoportrait sans visage et le caractère artificiel et pompeux de la toile de fond juxtaposés à l'univers enfantin du dessin donnent plus d'ampleur à la douleur ainsi exprimée. Ce sont toutes les expériences de rejet (parental, social et amoureux) vécues depuis l'enfance qui semblent convoquées ici. À l'exil associé à la condition de marginale, de dissidente, s'ajoute l'écartèlement que vit l'artiste entre le désir de domesticité entre lesbiennes et le besoin de dénoncer un système qui rend la chose difficile, voire impossible. Toutefois, devant les violences que s'impose l'artiste (dans Self Portrait/Pervert - 1994, Catherine Opie pose avec les bras transpercés par vingt-huit aiguilles, un perçage au mamelon droit et le mot « Pervert » découpé dans la chair de son torse) et le succès que son art remporte auprès des grandes institutions, on se demande si l'idéologie dominante et son système de valeurs ne finissent pas par l'emporter.

\section{Le Sang retrouvé}

Parallèlement aux approches centrées sur le corps et ses souffrances, il en est qui investissent des formes évocatrices du pouvoir et des façons dont il s'exerce pour le dénoncer. Dans ce cas, le sujet du sang peut être traité de manière beaucoup plus indirecte ou métaphorique. L'Américaine Barbara Kruger associe mots et images chocs ${ }^{6}$ en jouant, par exemple, de la force d'impact symbolique que possèdent le noir, le blanc et le rouge écarlate: "In her works, écrit Juliana Enberg, [...] power is interrogated and interpreted through the social, economic and political arrangements which motor the life impulses of love, hate, sex and death" $"$.

Dans une œuvre sans titre (1982), la photographie en noir et blanc 
d'une main abîmée et pansée est barrée à la manière des panneaux d'interdiction par un trait rouge oblique (/) arborant les mots « We will » en blanc. En bas de la photo, une bande horizontale et blanche porte cette fois les mots « undo you » en gros caractères noirs. Nous pouvons ainsi choisir de lire ces deux énoncés séparément ou à la suite l'un de l'autre. Lus séparément, ils semblent banals, sans grande portée comme une marque de jeans ou un slogan à la mode. Lus ensemble, ils acquièrent une toute autre dimension, révèlent une volonté de destruction du « lecteur » (circonstanciel et générique) fort déroutante, à ce point déroutante qu'on a du mal à croire que ces énoncés soient mis en présence l'un de l'autre. Ce trouble est accentué par le jeu des couleurs qui oppose l'innocence de la volonté (les mots «We will » sont en blanc) et la charge de l'injonction (les mots « undo you » sont en noir), ainsi que par le traitement objectif de l'image composée de photos d'archives ou scientifiques, et donc présentant des faits, la réalité derrière les (beaux) discours. Il ne manque que le sang qu'évoque fortement la barre oblique apparaissant en travers de l'image comme une blessure, une plaie.

Une autre œuvre de Kruger mérite également qu'on s'y attarde. On y voit le reflet du visage d'une femme en pleurs dans un miroir brisé. Un projectile a heurté la glace à la hauteur du front. La photographie englobe une des mains de la femme dont les doigts, aux ongles vernis, tiennent un fragment du miroir. L'image est coiffée des mots " you are " et bordée en bas du mot " yourself ». Chaque lettre est un gros caractère noir placé dans un carré blanc. Chaque carré est de guingois par rapport aux autres comme si on avait secoué tout l'ensemble. Au centre de l'image apparaît en tout petit et en rouge le mot « not » qui annule le sens que formerait l'enchaînement des énoncés " you are " et " yourself ». La femme a-t-elle vu la balle avant que celle-ci ne l'atteigne ou fracasse le miroir? La phrase «You are not yourself " semble dire à la " gent féminine »: attention aux miroirs, ils tuent l'être.

Également hantée par les manipulations idéologiques et le sort réservé aux femmes, l'artiste new-yorkaise Kiki Smith présente des œuvres plus troublantes encore, car le corps féminin est représenté dans sa matérialité la plus crue et sans aucune concession. Il apparaît à moitié accroupi avec une série de longs rubans de perles de verre écarlates s'échappant du vagin à la manière d'une traîne de robe de mariée (Untitled, Train 1994), recroquevillé sur le sol, rougi et ouvert tout le long de la colonne vertébrale qui prend l'allure d'une vulve suturée (Blood Pool 1992), ou littéralement écorché (Virgin Mary 1992) et 
percé aux poignets d'où coule de l'eau comme s'il était vidé de sa substance (Standing 2001). Autrement, le corps dépossédé est évoqué par de fortes images comme dans l'installation intitulée Bloodline (1995) où l'artiste a mis à la suite de l'autre 100 unités en verre soufflé rubis possédant la forme de globules rouges.

On ne saurait clore cette section sans mentionner le travail de Martha Fleming et Lyne Lapointe. Également intéressées par l'analyse des discours, elles se réfèrent aussi aux contes, légendes et mythes, accordent une attention particulière à l'organique et à la nature (animaux et végétaux) et intègrent/réhabilitent des approches et des médiums artisanaux. Particulièrement attentives aux pratiques de guérison et de plaisir, elles infusent ludisme et sensualité à leurs œuvres. D'où les petits bonheurs formels qui émaillent Eat $\mathrm{Me} / \mathrm{Drink}$ me/ Love me, installation inspirée par la poésie d'Emily Dickinson et de Christina Rossetti. Le titre de l'œuvre renvoie d'ailleurs au passage le plus homo-érotique du poème Goblin Marquet, qui présente un portrait inquiétant $\mathrm{du}$ traitement que nos sociétés réservent aux femmes. La pièce «Sangsue/ Mandragore » sur laquelle je m'attarderai maintenant rappelle le côté le plus noir de ce traitement.

L'œuvre consiste en un morceau creux d'écorce au centre duquel se trouve un dessin de la mandragore, cette racine en forme de corps humain. Comme dans la majorité des gravures représentant cette plante à laquelle on accorde des vertus médicinales, le visage est féminin. Évocatrice d'un corps de femme par sa rondeur, son ouverture et la présence d'un petit " être " en son centre, l'écorce est transpercée par des branches et suspendue au milieu d'un cadre orné de dessins représentant un serpent, un scorpion, une fourmi, un rat, une tarentule et une sangsue, toutes des bestioles qui piquent, pénètrent et/ou inspirent le dédain. Visuellement, l'œuvre peut aussi rappeler une déchirure, des points de suture ou une séance de dissection. En entrevue, Lyne Lapointe m'apprenait qu'une des tortures qui furent infligées aux femmes consistait à faire pénétrer des fourmis rouges dans leur vagin. Dans Eat $\mathrm{Me} /$ Drink $\mathrm{Me} /$ Love $\mathrm{Me}$, les deux artistes ne se contentent pas toutefois $d$ 'honorer la mémoire de celles dont le sang a coulé injustement, elles ont " recueilli » en quelque sorte ce sang en prenant exemple sur leurs vies et celles de toutes les artistes qui ont créé malgré les difficultés et les épreuves. De la sorte, elles perpétuent l'hommage que rend le poème de Rossetti à la sororité: 
For there is no friend like a sister,

In calm or stormy weather,

To cheer one on the tedious way,

To fetch one if one goes astray,

To lift one if one totters down,

To strengthen whilst one stands. (14)

$D^{\prime}$ autres auteures ${ }^{8}$ et artistes visuelles ${ }^{9}$ pourraient être signalées. Dans les oeuvres que nous avons citées, nous trouverions des représentations dissidentes du sang, de figures et d'images existantes (photos d'archives, écorchés de manuels de médecine, mythe de l'Eucharistie). Dans la mesure où ces références sont comprises par tous, elles forment un alphabet de représentations qui devient incontournable. Fortes de l'expérience de leurs aînées, ces féministes utilisent toutefois ces références avec précaution. Comme Lizzie, dans Goblin Market, il s'agit de prendre le fruit défendu sans y goûter. De la sorte, elles peuvent en "servir » tout juste la quantité qu'il faut pour opérer un déplacement de perspective et ainsi faire apparaître l'envers du décor ou les coulisses de ce que l'on appelle la réalité.

Nous venons de voir que des auteures et des artistes ont mis au jour le caractère sexospécifique des représentations du sang. Leur appropriation de ces représentations comme matière première, littéralement et conceptuellement, leur a permis d'en révéler les limites et le caractère biaisé. Dans leurs travaux et œuvres, la perception du sang menstruel comme signe d'un manque/ment des femmes devient plutôt celui d'une lacune au niveau du savoir, du savoir que l'on possède sur ce type de sang et du savoir que l'on rend accessible. De même, les figures de femmes comme victimes naturelles ("Tu enfanteras dans la douleur »), comme agneaux sacrificiels nécessaires au bien de tous et justifiant l'existence de "protecteurs » de l'ordre moral, collectif, familial, etc., autant dire de bourreaux, sont lues comme indices d'une oppression historique, comme mesures punitives exemplaires, et ne possédant par conséquent qu'un fondement idéologique. Enfin, les assertions du type « les femmes n'aiment pas le sang " témoignent quant à elles du conditionnement qui s'exerce sur ce groupe social, puisqu'elles tombent dès que ces dernières accèdent à une certaine liberté.

En ce sens, c'est toute la question du contrôle de ces représentations et des intérêts qu'elles servent que soulèvent les féministes face aux représentations du sang dans les sociétés occidentales. Car que 
l'on soit femme ou homme, blanc ou noir, petit ou grand, pauvre ou riche, le sang qui coule dans nos veines est exactement le même.

Notes

1 Voir la bibliographie de Paula Wansbrough, et Kathleen O'Grady sur le site: //www.mith2.umd.edu WomensStudies/Bibliographies/menstruation.

2 Selon l'Organisation mondiale de la santé, 2 millions de fillettes et adolescentes sont sexuellement mutilées chaque année dans 28 pays. On compte actuellement 130 millions de femmes excisées dans le monde. Selon le Rapport du Fonds des Nations Unies pour la population (FNUAP), une femme est battue toutes les 15 secondes aux USA. Au Pakistan, au moins 1000 femmes ont été assassinées l'an dernier pour venger 1 ' « honneur masculin ». Toujours selon le FNUAP, au moins 4 millions de femmes et de fillettes sont achetées et vendues annuellement dans le monde entier à de futurs époux, à des proxénètes ou à des marchands d'esclaves.

${ }^{3}$ Voir entre autres Les Dames galantes de Brantôme.

4 « Oui, la violence féminine existe. Et elle est de plus en plus préoccupante parce qu'en hausse importante chez les adolescentes, " Katia Gagnon, " Le côté noir des femmes ".

${ }^{5}$ Intitulée Vanitas: Flesh Dress for an Albino Anorectic, cette robe, confectionnée à partir de 50 livres de tranches de bifteck en décomposition, est accompagnée de la photographie d'une femme qui la revêt. L'œuvre a d'abord été exposée au Musée des Beaux-arts du Canada en 1987. 6 Parmi les phrases représentées, on compte celles-ci: « Do I have to give up me to be loved by you? ", "Love for sale ", " How dare you not be me? »

${ }^{7}$ Site: www.geocities.com/SoHo/Cafe/9747/kruger.html, entretenu par niina (niina@zip.com.au).

8 Je pense, par exemple, aux journalistes correspondantes de guerre comme les Américaines Jannet Flaner et Nancy Cunard en Europe ou Judith Jasmin, ici. Et je n'oublie pas que Gertrude Stein et Alice Toklas se sont faites ambulancières.

${ }^{9}$ On trouvera sur l'Internet au site de Tania Huerta, mentionné plus haut, d'autres noms d'artistes comme ceux de Judy Chicago, Marina Abramovic ou Carolee Scheenmann. 


\section{Bibliographie}

Angier, Nathalie, Woman. An Intimate Geography. New York: Random House, 1999. Bersianik, Louky. L'Eugélionne, Montréal: La Presse, 1976.

Brant, Peter. "An interview with Sue Coe », The RyeCatcher. Revolution of the mind through inspiration of the soul. http://www.theryecatch er.com/scoe.html

Brantôme. Les Dames galantes. Paris: Éditions Garnier Frères, 1960.

Caputi, Jane. "On Psychic Activism: Feminist Mythmaking ». The Woman's Companion to Mythology (sous la direction de Carolyne Larrington). Londres: Harper Collins Publisher, 1997.

Clark, Edward H., Sex in Education; or A Fair Chance for the Girls, 1883. Cité par Mary Roth Walsh dans Doctors Wanted: No Women Need Apply. New Haven: Yale University Press, 1997.

Coe, Sue, Dead Meat Book. New York et Londres: Four Walls Eight Windows, 1996. Dowling, Colette, The Frailty Myth. Women Approaching Physical Equality. New York: Random House, 2000,

Falini, Daniela. «Kahlo's disturbing art » dans « Frida Kahlo \& contemporary thoughts ", www.fridakahlo.com

Furguson, Russell. " "How I think": An interview with Catherine Opie ». The Photographer's Gallery. London: 2000.

Gagnon, Katia. « Le côté noir des femmes». La Presse (vendredi 8 mars 2002). cyberpresse.ca.

Graves, Robert, The White Goddess: A Hystorical Grammar of Poetic Myth. Londres: Faber, 1948,

Heller, Nancy G. Women Artists: An Illustrated History. New York: Abbeville Press, 1997.

Huerta, Tania. Blood in Context. htttp://citd.scar.utoronto.caVPABO4 /projects/Tania_Huerta/pages/index.html

Kramarae, Cheris et Paula A. Treichler,. Feminist Dictionary. Boston, Londres et Henly: Pandora, 1985.

Luc, Pierre. «Plus de pitié pour les meurtrières », editeur@lejournal2000.cjb.net La Nouvelle Evangile.

Rossetti, Christina. Goblin Marquet. http://www.crokercom/ lwn/goblinhtml Walker, Barbara G. The Woman's Encyclopedia of Myths and Secrets. San Francisco: Harper\&Row, 1983.

Wittig, Monique. Le Corps lesbien. Paris: Minuit, 1973.

—. etSandeZeig. Broullon pour un dictionnaire des amantes. Paris: Grasset, 1976. 